\title{
Cosmic Dynamics of Bose-Einstein Condensates
}

\author{
James E. Lidsey ${ }^{1}$ \\ Astronomy Unit, School of Mathematical Sciences, \\ Queen Mary, University of London, Mile End Road, LONDON, E1 4NS, U.K.
}

\begin{abstract}
A dynamical correspondence is established between positively curved, isotropic, perfect fluid cosmologies and quasi-two-dimensional, harmonically trapped BoseEinstein condensates by mapping the equations of motion for both systems onto the one-dimensional Ermakov system. Parameters that characterize the physical properties of the condensate wavepacket, such as its width, momentum and energy, may be identified with the scale factor, Hubble expansion parameter and energy density of the universe, respectively. Different forms of cosmic matter correspond to different choices for the time-dependent trapping frequency of the condensate. The trapping frequency that mimics a radiation-dominated universe is determined.
\end{abstract}

PACS NUMBERS: 98.80.Cq, 03.75.Kk

${ }^{1}$ Electronic mail: J.E.Lidsey@qmul.ac.uk 
In recent years, analogies between various condensed matter systems and different branches of gravitational physics have been developed. (For reviews, see, e.g., Refs. 1, 2]). For example, the propagation of acoustic waves in an irrotational, inviscid, barotropic fluid is formally equivalent to that of a massless scalar field on a curved, Lorentzian spacetime [3. This implies that quantum field theory in curved space can be modeled in terms of the quantization of the sound wave (phonon field). Furthermore, it is possible to model a black hole acoustically in terms of supersonic fluid flow and, in principle, quantum effects associated with black hole event horizons may then be studied within the context of condensed matter configurations [3, 4, 5]. A related phenomenon is that of cosmological particle production arising in a time-dependent gravitational field and the description of such a process in a dynamical Bose-Einstein condensate has recently been investigated [6, 7, 8, 9].

Identifying a link in this way between gravitational and non-gravitational physical systems is of great importance. To date, attention has focused primarily on the kinematical properties of general relativity, such as the existence of event horizons. The purpose of this work is to note that a dynamical correspondence may also be established between isotropic, four-dimensional cosmological models and harmonically trapped, quasi-two-dimensional Bose-Einstein condensates. The correspondence arises because the equations of motion for both systems can be mapped onto the one-dimensional Ermakov system [10, 11]. Ermakov systems arise in a variety of physical phenomena [12, 13, 14] and the one-dimensional case corresponds to a second-order, non-linear, ordinary differential equation (ODE) 10, 11.

We begin by considering the condensed matter system. A Bose-Einstein condensate is the ground state of a collection of interacting bosons trapped by an external potential. In the limit where the number of atoms is sufficiently large and the atomic interactions are sufficiently weak, the mean-field approximation may be invoked, where the effect felt by a particular atom due to the ensemble is approximated by the mean action of the entire fluid on the particle. (For a review, see, e.g., Ref. [15]). In this case, the macroscopic wavefunction for the condensate, $\Psi$, is determined by the Gross-Pitaevskii equation [16]

$$
i \hbar \frac{\partial \Psi}{\partial t}=-\frac{\hbar^{2}}{2 m} \nabla^{2} \Psi+V(\mathbf{r}, t) \Psi+g|\Psi|^{2} \Psi
$$

where $m$ is the mass of the atoms in the condensate, $V(\mathbf{r}, t)$ represents the trapping potential, and $g$ parametrizes the strength of the atomic interactions.

Eq. (11) is formally equivalent to a three-dimensional, non-linear Schrödinger equation and, in general, it is non-integrable. However, in two dimensions it is possible to determine the dynamics of the wavefunction by employing the "moment method' [17, 19. In this approach, integral relations are constructed directly from the wavefunction without solving the Schrödinger equation explicitly and the evolution of these physical quantities then parametrizes the dynamics of the wavepacket.

In view of this, we consider a two-dimensional, cylindrically symmetric BoseEinstein condensate in a parabolic trapping potential $V(r, t)=m \omega^{2} r^{2} / 2$ with a 
time-dependent frequency, $\omega=\omega(t)$. Lower-dimensional Bose gases are currently of interest both from the theoretical [20, 21, 19, 22] and observational perspectives and two-dimensional condensates have recently been observed experimentally [23. A Bose-Einstein condensate becomes effectively two-dimensional when the excitations in a given direction are frozen and this occurs when the confining frequency in that direction is significantly greater than the energy for the thermal fluctuation.

We now briefly summarize the moment method [17, 18, 19]. For the case of a constant interaction, $g$, the four integral parameters [17, 19]

$$
\begin{array}{r}
I_{1}(t)=\int d^{2} x|\Psi|^{2} \\
I_{2}(t)=\int d^{2} x r^{2}|\Psi|^{2} \\
I_{3}(t)=i \int d^{2} x r\left[\Psi \frac{\partial \Psi^{*}}{\partial r}-\Psi^{*} \frac{\partial \Psi}{\partial r}\right] \\
I_{4}(t)=\frac{1}{2} \int d^{2} x\left[|\nabla \Psi|^{2}+g|\Psi|^{4}\right]
\end{array}
$$

are defined, where it is assumed that $\Psi=\Psi(r, t)$ and we have specified $\hbar=m=1$ without loss of generality. Eqs. (2) - (5) admit physical interpretations in terms of the norm, width, radial momentum and energy of the wavepacket, respectively [19.

By differentiating Eqs. (2)-(5) in turn and substituting in Eq. (11), it can be shown that these integral parameters satisfy the set of coupled, first-order, ODEs 19]:

$$
\begin{array}{r}
\frac{d I_{1}}{d t}=0 \\
\frac{d I_{2}}{d t}=I_{3} \\
\frac{d I_{3}}{d t}=-2 \omega^{2}(t) I_{2}+4 I_{4} \\
\frac{d I_{4}}{d t}=-\frac{1}{2} \omega^{2}(t) I_{3} .
\end{array}
$$

It may now be verified by direct differentiation that the quantity

$$
Q \equiv 2 I_{4} I_{2}-\frac{I_{3}^{2}}{4}
$$

is a constant and defining $X \equiv I_{2}^{1 / 2}$ then implies that the system (7)-(10) is solved by solving the non-linear differential equation

$$
\frac{d^{2} X}{d t^{2}}+\omega^{2}(t) X=\frac{Q}{X^{3}}
$$


Eq. (11) corresponds to the one-dimensional Ermakov system and is known as the Pinney (or Ermakov-Pinney) equation [10, 11]. The general solution of Eq. (11) is given by 11 .

$$
X=\left[A X_{1}^{2}+B X_{2}^{2}+2 C X_{1} X_{2}\right]^{1 / 2},
$$

where $X_{1,2}(t)$ are linearly independent solutions to the equation

$$
\frac{d^{2} X_{i}}{d t^{2}}+\omega^{2}(t) X_{i}=0
$$

the constants $\{A, B, C\}$ satisfy the constraint

$$
A B-C^{2}=\frac{Q}{W^{2}}
$$

and

$$
W \equiv X_{1} \frac{d X_{2}}{d t}-X_{2} \frac{d X_{1}}{d t}
$$

is the Wronskian. It follows, therefore, that the evolution of the wavepacket for a given trapping frequency, $\omega^{2}(t)$, is fully determined by solving the linear oscillator equation (13).

We now proceed to consider cosmological dynamics. Our aim is to identify a class of models where the expansion (or contraction) of the universe can be represented as an Ermakov system. Recently, it was shown that the Einstein field equations for a spatially flat, isotropic and homogeneous Friedmann-Robertson-Walker (FRW) universe can be reduced to an Ermakov-Pinney equation when the matter is a mixture of (non-interacting) perfect fluids [13]. However, in this case, the sign of the nonlinear term in the Ermakov-Pinney equation is opposite to that of Eq. (11). This difference in sign arises because the energy densities of the matter fields are assumed to be positive-definite.

In view of this, we consider the effects of introducing spatial curvature into the analysis. Einstein's field equations for the spatially curved FRW cosmology with perfect fluid matter are given by

$$
\begin{aligned}
H^{2}= & \frac{1}{a^{2}}\left(\frac{d a}{d \tau}\right)^{2}=\frac{2}{3} \rho-\frac{k}{a^{2}} \\
& \frac{d \rho}{d \tau}+3 H(\rho+p)=0,
\end{aligned}
$$

where $H(\tau)$ is the Hubble parameter, $a(\tau)$ is the scale factor of the universe, $\tau$ represents cosmic (proper) time, $k$ is the curvature constant and units are chosen such that Newton's constant is given by $4 \pi G=1$. The pressure and energy density of the matter are given by $p(\tau)$ and $\rho(\tau)$, respectively. In principle, Eqs. (16) and (17) are solved once the equation of state, $p=p(\rho)$, has been specified. Eq. (17) follows as a direct consequence of energy-momentum conservation. 
Eqs. (16) and (17) may be expressed as an Ermakov system by interpreting the matter source as a self-interacting scalar field, $\phi=\phi(\tau)$, that is minimally coupled to Einstein gravity. Formally, this is equivalent to expressing the energy density and pressure as

$$
\rho=\frac{1}{2}\left(\frac{d \phi}{d \tau}\right)^{2}+U(\phi), \quad p=\frac{1}{2}\left(\frac{d \phi}{d \tau}\right)^{2}-U(\phi),
$$

where $U(\phi)$ represents the potential energy associated with the self-interactions of the scalar field. Differentiating Eq. (16) with respect to cosmic time and substituting Eqs. (17) and (18) then implies that

$$
\frac{1}{a} \frac{d^{2} a}{d \tau^{2}}-\frac{1}{a^{2}}\left(\frac{d a}{d \tau}\right)^{2}=-\left(\frac{d \phi}{d \tau}\right)^{2}+\frac{k}{a^{2}}
$$

and defining a new independent variable

$$
\frac{d}{d \tau}=a \frac{d}{d t}
$$

transforms Eq. (19) into the Pinney equation:

$$
\frac{d^{2} a}{d t^{2}}+\left(\frac{d \phi}{d t}\right)^{2} a=\frac{k}{a^{3}}
$$

A direct comparison between Eqs. (11) and (21) indicates that the dynamics of a positively curved $(k>0)$ FRW cosmology can be modeled in terms of a harmonically trapped Bose-Einstein condensate when cosmic time, $\tau$, is related to 'laboratory' time, $t$, through Eq. (20). The width of the wavepacket plays the same role as that of the cosmological scale factor:

$$
I_{2} \longleftrightarrow a^{2}
$$

and it follows from Eqs. (17) and (20) that the radial momentum of the wavepacket is associated with the expansion velocity of the universe:

$$
I_{3} \longleftrightarrow 2 \frac{d a}{d \tau}
$$

This implies that the Hubble parameter may be identified with the combination

$$
H^{2} \longleftrightarrow \frac{1}{4} \frac{I_{3}^{2}}{I_{2}}
$$

and consequently the comoving Hubble radius is determined by the inverse of the wavepacket momentum:

$$
\frac{1}{a H} \longleftrightarrow \frac{2}{I_{3}}
$$




\begin{tabular}{||c|c||}
\hline \hline Cosmological Parameter & Wavepacket Parameter \\
\hline$a$ & $I_{2}^{1 / 2}$ \\
$H^{2}$ & $I_{3}^{2} /\left(4 I_{2}\right)$ \\
$\rho$ & $3 I_{4}$ \\
$p$ & $I_{2} \omega^{2}-3 I_{4}$ \\
\hline \hline
\end{tabular}

Table 1: The dynamical correspondence between a positively curved FRW cosmology with a perfect fluid matter source and a harmonically trapped, quasi-two-dimensional Bose-Einstein condensate. The width, radial momentum and energy of the wavepacket are analogous to the scale factor of the universe, the Hubble parameter and energy density of cosmic matter, respectively. The cosmic dynamics is determined once the relationship between the pressure and energy density has been specified. This is equivalent in the condensed matter context to choosing the time dependence of the trapping potential.

Furthermore, substituting Eqs. (22) and (24) into Eq. (10) and comparing the result with the Friedmann equation (16) implies that the energy of the condensate wavepacket corresponds to the energy density of cosmic matter

$$
I_{4} \longleftrightarrow \frac{\rho}{3}
$$

where we identify $Q=k$. Finally, the kinetic energy of the scalar field is related to the trapping frequency of the condensate such that

$$
\left(\frac{d \phi}{d \tau}\right)^{2} \longleftrightarrow I_{2} \omega^{2}
$$

and this implies that the pressure of the field is determined by

$$
p \longleftrightarrow I_{2} \omega^{2}-3 I_{4}
$$

Thus, the width, radial momentum and energy of the condensate wavepacket can be identified with a corresponding cosmological parameter. This correspondence is summarized in Table 1. Eq. (10) may now be viewed as a novel way of expressing the Friedmann equation in terms of properties of the wavefunction. Moreover, by employing the correspondences in Table 1, we deduce that Eq. (9) is equivalent to the conservation equation (17).

It is of interest to consider specific classes of cosmological models and their condensate analogues. For example, inflationary solutions are of particular importance to early universe cosmology. The condition for inflationary expansion is that the scale factor of the universe should accelerate (with respect to cosmic time) and it is straightforward to verify that this condition is satisfied whenever the radial momentum of the wavepacket grows:

$$
\frac{d^{2} a}{d \tau^{2}}>0 \quad \Longleftrightarrow \quad \frac{d I_{3}}{d t}>0
$$


Another important class of models is characterized by the barotropic equation of state, $p=(\gamma-1) \rho$, where $0 \leq \gamma \leq 2$ is a constant. In this case, the correspondence in Table 1 implies that the trapping potential satisfies $\omega^{2}=3 \gamma I_{4} / I_{2}$ and substituting this condition into Eq. (9) and integrating implies that

$$
I_{4}=\frac{A}{I_{2}^{3 \gamma / 2}}, \quad \omega^{2}=\frac{3 \gamma A}{I_{2}^{(2+3 \gamma) / 2}},
$$

where $A$ is an arbitrary integration constant. A further substitution of Eqs. (7) and (30) into Eq. (10) then yields a first-order differential equation for the width of the wavepacket:

$$
\frac{d I_{2}}{d t}=2 \sqrt{2 A I_{2}^{(2-3 \gamma) / 2}-1}
$$

where we have specified $Q=1$ without loss of generality. Eq. (31) may be integrated to yield the solution

$$
\begin{aligned}
{ }_{2} F_{1}\left[\frac{1}{2}\left(\frac{3 \gamma+2}{3 \gamma-2}\right), \frac{1}{2} ; \frac{1}{2}\left(\frac{9 \gamma-2}{3 \gamma-2}\right) ; \frac{1}{2 A}\right. & \left.I_{2}^{(3 \gamma-2) / 2}\right] I_{2}^{(3 \gamma+2) / 4} \\
& =\sqrt{\frac{A}{2}}(3 \gamma+2) \Delta t
\end{aligned}
$$

where ${ }_{2} F_{1}$ is the hypergeometric function [24], $\Delta t \equiv t-t_{0}$ and $t_{0}$ represents the second integration constant.

Eqs. (30) and (32) together determine the time dependence of the condensate trapping potential that mimics a cosmology with a given equation of state, $\gamma$. The limiting case of $\gamma=0$ represents a universe dominated by a cosmological constant and corresponds to a vanishing trapping potential. On the other hand, a universe dominated by relativistic matter has an equation of state given by $\gamma=4 / 3$ and in this case Eq. (32) simplifies to

$$
\begin{array}{r}
\sin ^{-1} \sqrt{x}-\sqrt{x-x^{2}}=\frac{1}{A} \Delta t \\
x \equiv \frac{(4 A)^{1 / 3}}{2 A} \frac{1}{\omega^{2 / 3}} .
\end{array}
$$

Eq. (32) also simplifies for a universe dominated by pressureless matter $(\gamma=1)$ :

$$
\begin{array}{r}
3 \sin ^{-1} \sqrt{y}-(3+2 y) \sqrt{y-y^{2}}=\frac{1}{A^{2}} \Delta t \\
y \equiv \frac{(3 A)^{1 / 5}}{2 A} \frac{1}{\omega^{2 / 5}} .
\end{array}
$$

Although Eqs. (33) and (34) can not be inverted analytically, the explicit time dependence of the trapping potential can be expressed in a closed form for a 'stiff' perfect 
fluid $(\gamma=2)$. We find in this case that

$$
\begin{aligned}
& I_{2}=\left[2 A-4(\Delta t)^{2}\right]^{1 / 2} \\
& \omega^{2}=\frac{3 A}{2\left[A-2(\Delta t)^{2}\right]^{2}} .
\end{aligned}
$$

To summarize thus far, it has been shown that positively curved, perfect fluid FRW cosmologies can be modeled dynamically in terms of quasi-two-dimensional Bose-Einstein condensates, where there exists a one-to-one correspondence between the type of matter in the universe and the functional form of the time-dependent trapping potential of the condensate. The physical properties of the wavefunction can be identified with the fundamental cosmological parameters.

It is of interest to consider extensions of the correspondence to three-dimensional condensates. The moment method as outlined above can not be applied in three dimensions unless certain approximations are made [18. However, an alternative approach is to employ the well known scaling properties that are exhibited by the wavefunction under the evolution of a time-dependent confining potential [20, 21, 25]. In this approach, the ansatz

$$
\Psi=\frac{1}{b^{3 / 2}} \chi \exp \left[\frac{i}{2} \frac{b_{t}}{b} r^{2}\right]
$$

is made, where $b=b(t), \chi=\chi(t, r)$ and a subscript denotes differentiation with respect to laboratory time, $t$ [20]. Substituting Eq. (36) into the non-linear Schrödinger equation (11) and defining new variables

$$
\sigma \equiv \frac{r}{b} \quad \theta \equiv \int \frac{d t}{b^{2}}
$$

then implies that Eq. (11) reduces to

$$
\begin{array}{r}
\frac{i}{b^{2}} \frac{\partial \chi}{\partial \theta}+\frac{1}{2 b^{2}} \nabla_{\sigma}^{2} \chi-\frac{g}{b^{3}}|\chi|^{2} \chi-\frac{1}{2} \frac{\omega_{0}^{2} \sigma^{2} \chi}{b^{2}} \\
=\frac{1}{2} r^{2} \chi\left[\frac{1}{b} \frac{d^{2} b}{d t^{2}}+\omega^{2}(t)-\frac{\omega_{0}^{2}}{b^{4}}\right],
\end{array}
$$

where $\omega_{0}$ is a constant and the last terms on both sides of Eq. (38) have been introduced by hand.

If the interaction strength, $g$, is time-independent, it is not possible in general to separate the left and right hand sides of Eq. (38). On the other hand, the scattering length may be varied by employing a magnetic field induced Feshbach resonance [26]. If the interaction is tuned such that $g(t) \propto b(t)$, all terms on the left-hand side of Eq. (38) scale as $b^{-2}$ and, in this case, Eq. (38) separates when $b(t)$ satisfies the Ermakov-Pinney equation

$$
\frac{d^{2} b}{d t^{2}}+\omega^{2}(t) b=\frac{\omega_{0}^{2}}{b^{3}}
$$


It then follows that the rescaled wavefunction, $\chi$, formally satisfies the same nonlinear Schrödinger equation as the original wavefunction but with a constant trapping frequency and interaction strength. Comparing Eqs. (21) and (39) implies that the scaling parameter, $b$, may be identified with the scale factor of the universe, where laboratory time is again related to cosmic time through Eq. (20).

We conclude by highlighting a number of questions that would need to be addressed in order to realise the above mathematical correspondences in a laboratory environment. In general, a sonic horizon may form in an expanding Bose-Einstein condensate, where the speed of the fluid exceeds the sound speed. Such an horizon forms due to an effective velocity field that increases with distance from the centre of the condensate [9. This could be problematic from a dynamical point of view since it may introduce physical effects into the system that may not be intrinsic to the cosmological model under consideration. In particular, ambiguities may arise when interpreting the analogue of cosmological particle production in a time-dependent gravitational field.

A key assumption that was made in establishing the correspondence between the condensate and cosmological systems was that the dynamics of the condensate wavefunction can be described in terms of the Gross-Pitaevskii equation (10) at each moment of time, i.e., that the configuration reacts instantaneously to changes in the trapping potential and scattering length of the atomic interactions. If this assumption is to remain valid, the majority of the atoms must remain in the condensate state (mean-field approximation) and the particle density and scattering length must be sufficiently small (dilute gas approximation). Moreover, to avoid complications with delays in the system, the timescales over which external parameters such as the trapping potential change should exceed the timescale for a pair of atoms to fully interact [9, 27]. Overall, these requirements imply that the trapping potential should change at a sufficiently slow rate. In this case, the correspondence (27) implies that only those cosmologies where the kinetic energy of the scalar field is sufficiently small could be simulated ${ }^{1}$.

A further important scale in the condensate is the 'healing' time, $t_{\text {heal }}$. The healing time is the characteristic timescale for variations in the wavefunction and plays an analogous role to that of the Planck time in cosmology. Comparison of cosmic and laboratory time through the correspondence (20) implies that $a d \tau=d t>t_{\text {heal }}$ and, for a cosmic timescale of the order of the Hubble time $d \tau=H^{-1}$ (representing the size of the cosmic horizon), this implies that $a>t_{\text {heal }} H$. Such considerations suggest that the correspondence we have developed could only be realised in practice for cosmological models satisfying a lower bound on the scale factor.

Nevertheless, one advantage of establishing correspondences between cosmology and condensed matter physics through Ermakov systems is that insight into the hidden symmetries of the two systems may be uncovered. For example, in the absence

\footnotetext{
${ }^{1}$ It should be noted, however, that in an inflationary context, the scalar field is necessarily rolling very slowly down its interaction potential.
} 
of a trapping potential, the action for a two-dimensional Bose-Einstein condensate described by the non-linear Schrödinger equation (11) is symmetric under a global $\mathrm{SL}(2, R)$ reparametrization of the time parameter: $\tilde{t}=(\alpha t+\beta) /(\gamma t+\delta)$, where $\{\alpha, \beta, \gamma, \delta\}$ are arbitrary constants satisfying the constraint $\alpha \delta-\beta \gamma=1$ [28, 29]. In general, this symmetry is broken by the inclusion of a time-dependent trapping potential. However, for the specific case where the potential varies as $\omega(t) \propto t^{-2}$, there exists a discrete duality symmetry with $\alpha=\delta=0$ and $\beta=-1 / \gamma[29$. This

implies that $\tilde{t}=-\beta^{2} / t$ and such a transformation maps an expanding solution onto a contracting one and vice-versa.

The associated cosmological model for this trapping potential may be deduced in the limit where the spatial curvature is dynamically negligible, as is the case during inflation. It follows from the correspondence summarized in Table 1 that for this trapping potential the scale factor has a power law dependence, $a(\tau) \propto \tau^{n}$ for some constant $n$, and the scalar field varies as $\phi(\tau) \propto \ln \tau$. This solution plays a central role in inflationary cosmology because it is one of the few models where the density and gravitational wave perturbation spectra can be calculated exactly without invoking the slow-roll approximation [30] (for a review, see, e.g., Ref. [31]). In effect, the solution is a scaling solution since the kinetic energy of the scalar field and the Hubble parameter decrease at the same rate. In view of its significance, it is interesting that this cosmological model is selected directly from symmetries exhibited by a class of Bose-Einstein condensates. It would be interesting to investigate whether an associated symmetry exists in the cosmological context as this would provide further insight into the density and gravitational wave perturbations generated during inflation.

Finally, an SL $(2, R)$ symmetry also arises in superstring-inspired inflationary cosmologies such as the pre-big bang scenario [32, where a duality symmetry relates contracting and expanding solutions. (For reviews, see, e.g., Ref. [33]). In these models, the dilaton and axion fields in the Neveu-Schwarz/Neveu-Schwarz sector of the string effective action parametrize the $\mathrm{SL}(2, R) / \mathrm{U}(1)$ coset and the existence of this symmetry implies that the density perturbations arising from fluctuations in these fields can be calculated exactly 34]. Furthermore, inflation in pre-big bang cosmology can be re-interpreted as the contracting phase of a universe dominated by a stiff perfect fluid [35] and this corresponds in the context of the present work to the condensate given in Eq. (35).

\section{Acknowledgments}

JEL is supported by the Royal Society. We thank C. Barceló, P. K. Ghosh and U. R. Fischer for helpful comments and communications. 


\section{References}

[1] Volovik G E 2001 Phys Rep 351195

Volovik G E 2003 The Universe in a Helium Droplet (Oxford: Oxford University)

[2] Novello M, Visser M and Volovik G E 2002 Artificial Black Holes (Singapore: World Scientific)

[3] Unruh W G 1981 Phys Rev Lett 461351

[4] Visser M 1998 Class Quantum Grav 151767

[5] Garay L J, Anglin J R, Cirac J I and Zoller P 2000 Phys Rev Lett 854643

Barceló C, Liberati S and Visser M 2001 Class Quantum Grav 181137

[6] Calzetta E A and Hu B L 2002 Preprint cond-mat/0207289

Calzetta E A and Hu B L 2002 Preprint cond-mat/0208569

[7] Fedichev P O and Fischer U R 2003 Preprint cond-mat/0303063

Fedichev P O and Fischer U R 2003 Preprint cond-mat/0304342

Fedichev P O and Fischer U R 2003 Preprint cond-mat/0307200

[8] Barceló C and Campos A 2002 Phys Lett B563 217

[9] Barceló C, Liberati S and Visser M 2003 Preprint gr-qc/0305061

Barceló C, Liberati S and Visser M 2003 Preprint cond-mat/0307491

[10] Ermakov V P 1880 Univ Izv Kiev 201

Milne W E 1930 Phys Rev 35863

[11] Pinney E 1950 Proc Amer Math Soc 1681

[12] Shahinpoor M and Nowinski J L 1971 Int J Nonlin Mech 6193

Goncharenko A M, Logvin Yu A, Samson A M and Shapovalov P S 1991 Opt Commun $\mathbf{8 1} 225$

Goncharenko A M, Logvin Yu A, Samson A M, Shapovalov P S and Turovets S I 1991 Phys Lett A160 138

Gaididei Yu B, Rasmussen K O and Christiansen P L 1995 Phys Rev E52 2951

Rasmussen K O, Gaididei Yu B, Bang O and Christiansen P L 1995 Math Comp Sim 40339

Hansen P L, Svensek D, Parsegian V A and Podgornik R 1999 Phys Rev E60 1956

Finelli F, Vacca G P and Venturi G 1998 Phys Rev D58 103514

Bertoni C, Finelli F and Venturi G 1998 Phys Lett A237 331

Finelli F, Gruppuso A and Venturi G 1999 Class Quantum Grav 163923 
Rosu H, Espinoza P and Reyes M 1999 Nuovo Cim B114 1435

Schief W K, Rogers C and Bassom A P 1996 J Phys A29 903

Berkovich L M and Rozov N H 1997 Arc Math (BRNO) 3375

Padilla P B E 2000 Preprint math-ph/0002005

[13] Hawkins R M and Lidsey J E 2002 Phys Rev D66 023523

[14] Williams F L and Kevrekidis P G 2003 Class Quantum Grav 20 L177

[15] Dalfovo F, Giorgini S, Pitaevskii L P and Stringari S 1999 Rev Mod Phys 71463

[16] Gross E P 1961 Nuovo Cim 20454

Pitaevskii L P 1961 Zh Eksp Teor Fiz 40646 [Sov Phys JETP 13 451]

[17] Belanger P A 1991 Opt Lett 16196

Porras M A, Alda J and Bernabeu E 1993 Appl Opt 325885

[18] Pérez-García V M, Porras M A and Vázquez L 1995 Phys Lett A202 176

García-Ripoll J J and Pérez-Garcia V M 1999 Preprint patt-sol/9904006

[19] García-Ripoll J J, Pérez-Garcia V M and Torres P 1999 Phys Rev Lett 831715

[20] Kagan Yu, Surkov E L and Shlyapnikov G V 1996 Phys Rev A54 R1753

[21] Pitaevskii L P and Rosch A 1997 Phys Rev A55 R853

[22] Petrov D S, Holzmann M and Shlyapnikov G V 2000 Phys Rev Lett 842551

Adhikari S K 2000 Phys Rev E62 2937

Lee M D, Morgan S A, Davis M J and Burnett K 2002 Phys Rev A65 043617

Khawaja U Al, Andersen J O, Proukakis N P and Stoof H T C 2002 Phys Rev

A66 013615

Haas F 2002 Phys Rev A65 033603

[23] Görlitz A 2001 Phys Rev Lett 87130402

Hammes M, Rychtarik D, Engeser B, Nägerl H C and Grimm R 2003 Phys Rev Lett 90173001

[24] Abramowitz M and Stegun I A (Eds.) 1964 Handbook of Mathematical Functions (New York: Dover Publications)

[25] Castin Y and Dum R 1996 Phys Rev Lett 775315

[26] Inouye S et al 1998 Nat 392151

Cornish S L et al 2000 Phys Rev Lett 851795

[27] Köhler T and Burnett K 2002 Phys Rev A65 033601 
[28] Ghosh P K 2002 Phys Rev A65 012103

Ghosh P K 2002 Phys Rev A65 053601

[29] Ghosh P K 2003 Phys Lett A308 411

[30] Lucchin F and Matarrese S 1985 Phys Rev D32 1316

Stewart E D and Lyth D H 1993 Phys Lett B302 171

[31] Lidsey J E, Liddle A R, Kolb E W, Copeland E J, Barreiro T and Abney M 1997 Rev Mod Phys 69373

[32] Gasperini M and Veneziano G 1993 Astropart Phys 1317

[33] Lidsey J E, Wands D and Copeland E J 2000 Phys Rep 337343 Gasperini M and Veneziano G 2003 Phys Rep 3731

[34] Copeland E J, Easther R and Wands D 1997 Phys Rev D56 874 Copeland E J, Lidsey J E and Wands D 1997 Nucl Phys B506 407

[35] Gasperini M and Veneziano G 1993 Mod Phys Lett A8 3701 